\title{
Joseph Uriot und die Gründung der öffentlichen Bibliothek des Herzogs Carl Eugen von Württemberg
}

In der Einleitung seiner Beschreibung der Feste, die im Februar 1764 stattfanden, erwähnt Joseph Uriot zum ersten Mal die Gründung einer öffentlichen Bibliothek: Der Herzog habe sie vor kurzem angekündigt. ${ }^{1}$ Uriot war sicher sehr froh, diese Nachricht zu verbreiten, denn er hatte die Anregung zu dieser Gründung gegeben. Anderthalb Jahre zuvor, am 27. September 1762, hatte er nämlich seinem Freund François Devaux geschrieben, er hoffe den Herzog Carl Eugen dazu zu bewegen, solch eine Anstalt zu öffnen: „j'espère determiner le duc dont je suis devenu le bibliothecaire avec cent Louis d'appointemens, à etablir une bibliotheque publique". ${ }^{2}$

\section{Als gebürtige Lothringer und gleichaltrig waren} Uriot und Devaux eng befreundet. Als Uriot seine Heimat verließ, um Schauspieler zu werden, begann ein regelmäßiger Briefwechsel. ${ }^{3}$ Niemandem außer Devaux hätte Uriot seine Hoffnung, die Gründung einer Bibliothek betreffend, anvertraut. Und niemandem sonst hätte er geschrieben, er wolle diese Anstalt leiten und dem Herzog seine eigene Büchersammlung verkaufen. Kann man aus diesem Verkaufsprojekt schließen, dass er in der Gründung nur seinen finanziellen Vorteil sah? Sicher nicht. Uriot war seit Jahren ein leidenschaftlicher Bücherliebhaber, der sich aber auch im Fach auskannte. Die Bücher seiner Sammlung hatte er sorgfältig ausgewählt. ${ }^{4}$ Und Bibliothekar zu sein, war für inn ein Beruf im vollen Sinne des Wortes.

Als er in Stuttgart ankam, hatte er diesen Beruf schon zweimal ausgeübt: zuerst in Lothringen, dann in Bayreuth. Nach seinem Studium an der Universität Pont-à-Mousson ${ }^{5}$ unterrichtete der hochgebildete Uriot Geographie und Geschichte an der Académie Royale des Gentilshommes-Cadets in Lunéville, einer Schule, die der polnische König Stanislas Leszczynski - damals Herzog von Lothringen - gestiftet hatte, um junge Adelige aus Lothringen und Polen gemeinsam zu erziehen. In dieser Schule war Uriot auch Bibliothekar. ${ }^{6} 1744$ stellte inn der Markgraf von Bayreuth als Schauspieler ein. Bald kamen noch die Verwaltung der markgräflichen Privatbibliothek ${ }^{7}$ und eine Lehrtätigkeit dazu: Uriot wurde Hauslehrer der Tochter des Markgrafen - die 1748 Carl Eugen heiratete - und Lehrer der Pagen. ${ }^{8}$

Bibliotheken und Schulen sind in Uriots Laufbahn eng verknüpft. Als Anhänger der Aufklärung, als Freimaurer, glaubte er an den Fortschritt der Menschheit. Zu diesem Fortschritt trugen Unterricht und Bücher in großem Maße bei. Dank der Gründung einer öffentlichen Bibliothek wäre der Zugang zur Wissenschaft höchst erleichtert. Das hat Uriot dem Herzog in seiner Rede am 13. Februar 1765 gesagt: „vous [...] formez une Bibliothèque Publique dans la quelle les Artistes \& les Gens de Lettres, ainsi que les Amateurs des Arts \& des Sciences trouveront en tout temps, pour cultiver leur goût \& pour augmenter leurs connoissances, des secours que la fortune des Particuliers ne leur permet presque jamais de se procurer." ${ }^{\text {9 }}$

1) Uriot, Joseph: Description des fêtes donnees à l'occasion du jour de naissance de son Altesse Serenissime Monseigneur le Duc Regnant de Wurtemberg et Teck, Stuttgart 1764, S. XIX-XX

2) Bibliothèque nationale de France, Manuskript NAF 15593. Die Stelle des Briefes, die die Bibliothek und Büchersammlung betrifft, ist im Anhang vollständig zitiert und übersetzt. In Zitaten sind die Schreibweise und die Zeichensetzung der Quellen beibehalten.

3) Devaux hat die Briefe lange behalten. Am 10. August 1786 sortierte er sie, verbrannte eine Menge und sendete den Rest einer Freundin, Mme Durival. Die Briefe wurden später verkauft, gehörten einst dem englischen Sammler Sir Thomas Phillipps und dann der BNF. Als Devaux sie seiner Freundin übermittelte, schrieb er, er habe viele Briefe vernichtet, obwohl sie wertvoll waren (Manuskript NAF 15593, BI. 1r: "d'un tres grand nombre que j'ay brulées, quoyque pleine[s] d'esprit, de raison et d'amitié").

4) Herrmann, Christian: Rarität und Ästhetik. Die Sammlung Uriot; in: Carl Eugens Erbe. 250 Jahre Württembergische Landesbibliothek, Stuttgart 2015, S. 46-51.

5) Pont-à-Mousson liegt zwischen Nancy und Metz. Herzog Karl III. von Lothringen hatte 1572 die Universität gegründet. Dort unterrichteten Jesuiten. Uriot war vorher schon ihr Schüler in Nancy gewesen.

6) Batz, August Friedrich: Beschreibung der Hohen Karls-Schule zu Stuttgart, 1783, S. 106 (WLB, Signatur: HB 7449).

7) Das schreibt am 23. Dezember 1746 Mme de Graffigny ihrem Freund Devaux. Uriot hatte "la garde de la biblioteque" (Correspondance de Madame de Graffigny, Bd. 8, hrsg. von Nicole Boursier, Oxford 2003, S. 178 - WLB, Signatur: 40/20169).

8) Die Pagen unterrichtete er in Geschichte. Dazu Heinritz, Johann Georg: Neue Beiträge zur Geschichte der Kreishauptstadt Bayreuth, Bayreuth 1839, S. 10

9) Uriot, Joseph: Discours prononcé à l'ouverture de la bibliothèque publique [...], Stuttgart 1765, S. 13. Das Wort "fortune" bedeutete "Schick sal", aber auch "Vermögen". In der deutschen Übersetzung der Rede steht "Glücks-Umstände" (S. 13). Uriot dachte vielleicht eher an "Vermögen", denn er wusste aus Erfahrung, was Bücher kosteten. 


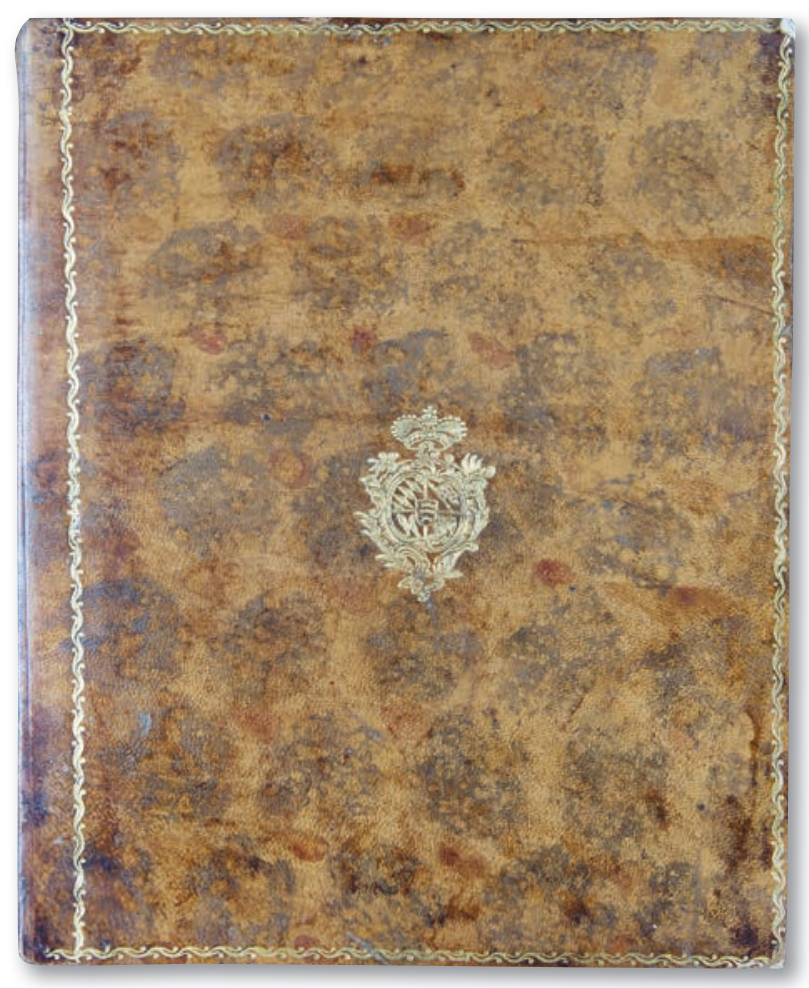

Abb. 1: Einband des „Discours sur la richesse et les avantages du duché de Würtemberg" (Bibliothèque Stanislas Nancy, Signatur: Rés. 10270)

Als Privatbibliothekar und Leser des Herzogs hatte Uriot Zugang zu inm. Er hat wahrscheinlich diese Gelegenheit genutzt, um inn zu überzeugen, eine öffentliche Bibliothek sei in seinem Staat unentbehrlich. Er konnte inm auch ein Vorbild nennen: die Bibliothek, die König Stanislas in Nancy am 28. Dezember 1750 gestiftet hatte. ${ }^{10}$ Diese Anstalt war mit einer Akademie - der Société Royale des Sciences et Belles-Lettres - verbunden. Die Mitglieder dieser Akademie - Gelehrte, Wissenschaftler - sollten die ihnen zugeschickten Werke prüfen und dann Preise austeilen. Sie sollten auch selbst zugunsten ihrer Mitmenschen nützliche Aufsätze schreiben. Eine Zeitschrift - "Mémoires de la Société Royale des Sciences et Belles-Lettres de Nancy" - wurde gegründet, um die besten Schriften zu veröffentlichen. ${ }^{11}$ Im "Stiftungs-Diplome der öffentlichen Bibliotheck" erwähnt der Herzog eine zukünftige Akademie, die der Société Royale gleicht: „Da bey Errichtung dieses Instituti Unser hauptendzweck dahin gehet, daß sich mit der Zeit in Unserer herzoglichen Residenz eine gelehrte Gesellschaft for-

10) Die Stiftungsurkunde wurde 1751 in Nancy gedruckt: Édit du Roy. Portant établissement d'une Bibliotéque publique à Nancy, \& Fondation de deux Prix (Bibliothèque Stanislas Nancy, Signatur: 702.046.8).

11) Im ersten Band der Zeitschrift (1754) wurden die Stiftungsurkunde und das Statut der Akademie wieder veröffentlicht (S. 3-12 und S. 83-104). Die Bibliothèque Stanislas bewahrt die Bände der Zeitschrift, die auch digitalisiert sind (BNF, Gallica).

12) Carl Eugen (Württemberg, Herzog): Stiftungs-Diplome der öffentlichen Bibliotheck, Stuttgart 1765, S. 14. Die besten Aufsätze sollten in der Hofdruckerei gedruckt und dann veröffentlicht werden (S. 15-16).

13) Uriot, Discours, S. 14-15.

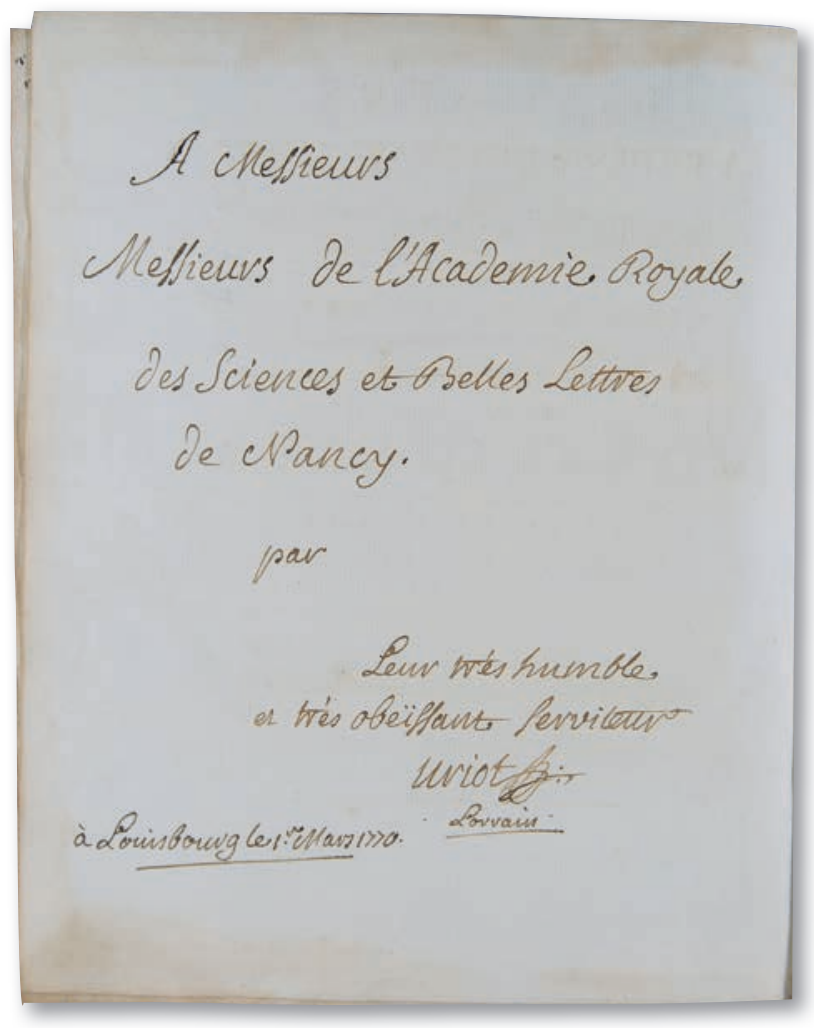

Abb. 2: Widmung im "Discours sur la richesse"

64

(31.) Le Cabinet d'Fiftoire naturelle, commenos par to Duc Évraro III, \& continué par le Duc Évrasto Loữs, ayant été, pour ainfi dire, anéanti dans plu-

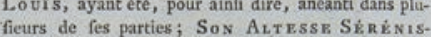
SIME fatisfaite des foins \& des lumières de $\mathrm{M}^{\mathrm{r}}$. le Profeffeur Vijcher, lainé, au quel il en a confié la grofeffeur a réfolu de donner fes ordres tant a la Fagarlé de Medecine, qu'aux Phyficiens du Pays de raffembler, \& d'y dépofer toutes les productions remarquables dans chacun des trois Règnes, qui doivent former cette Collettion,

Divers Ouvrages compofés par plufieurs MèdecinsNaturaliftes fur les curiofités naturelles qui fe trouvent dans le Würtemberg, nous apprennent qu'il y a peu de Pays qui en produife davantage, ni avec autant de varieté́; \& Mr. Raster, Profeffeur extra-ordinaire du Gymnafe de Stoutgard vient de finir un Ouvrage trè̀s curieux fur l'Hiftoire Naturelle du Pays fuivant le cours des rivières. axisososhon

(32.) MONSETGNEVR $L \mathrm{E}$ Due defirant de faire fleurir les Arts dans fes Etats, fonda en $77^{6} \mathrm{r}$, une Académie où dexcellents Artittes, a la téte des quels ente M. Dincter de la Garre de SoN RÉN1S-

Abb. 3: Korrekturvermerk Uriots

miren möge. So werden hiemit alle Liebhabere der Wissenschaften und Gelehrte eingeladen, sich sothanes Institutum zu Nuze zu machen, einander ihre Einsichten entweder mündlich, oder durch Briefwechsel mitzutheilen, und ihre Bemühungen, nach ihren Talenten auf Ausarbeitung solcher nüzlichen 
Werke, in welcher Sprache es seye, anzuwenden, wodurch überhaupt die Wissenschaften befördert und in Aufnahm gebracht werden, vornehmlich aber dem Vatterland Ehre und Vortheil zugehen möge". ${ }^{2}$ In seiner Rede sagt Uriot etwas Ähnliches. ${ }^{13}$ Das ist sicher kein Zufall.

Uriot wusste Bescheid über Königs Stanislas Zwillingsanstalten: Er reiste ab und zu nach Nancy ${ }^{14}$ und sein Freund Devaux war königlicher Leser und Mitglied der Société Royale. 1752 sendete Uriot der Akademie ein Gedicht, "L'éducation". Die besten Strophen wurden in der Zeitschrift namenlos veröffentlicht. Man weiß aber, dass er der Verfasser war. In der "Beschreibung der Hohen Karls-Schule" gehört nämlich das Gedicht zu seinen Schriften: „eine didaktische Ode, die in den Memoires der Akademie der schönen Künste und Wissenschaft zu Nancy zu lesen [ist]". ${ }^{15}$ Und er wollte zur Akademie als "correspondant" gehören. ${ }^{16}$ Kurz nach der Gründung der Bibliothek in Ludwigsburg sendete er deswegen nach Nancy einen Brief und den Sammelband "Fondation d'une bibliothèque publique par Son Altesse Sérénissime Monseigneur le duc régnant de Wurtemberg et Teck etc. le onze février MDCCLXV“. Der Einband war aus Kalbsleder: Carl Eugen hatte wahrscheinlich seine Genehmigung zur Sendung gegeben. ${ }^{17}$ Am 17. April 1765, während einer Sitzung, wurde Uriots Rede vom 13. Februar vorgelesen und bewundert. Jemand erinnerte sich aber plötzlich, dass er einst als bezahlter Schauspieler tätig gewesen war. Von seiner Kandidatur wurde dann nicht mehr geredet. ${ }^{18}$ Er war sicher enttäuscht und der Herzog vermutlich verärgert: man hatte nämlich sein Werk etwas verachtet.

Am 1. März 1770 schickte Uriot der Akademie, mit einer handschriftlichen Widmung, die Rede, die er am 11. Februar gehalten hatte, „Discours sur la richesse et les avantages du duché de Würtemberg". Auf dem Vorderdeckel des schönen Einbandes sind die Wappen der Herzöge von Württemberg goldgeprägt ${ }^{19}$. War es Uriots und Carl Eugens gemeinsame Antwort an die etwas starrsinnigen Mitglieder der Akademie?

\section{Quellenfunde zu Uriot \\ BNF Paris, Manuskript NAF 15593, BI. 23v: Auszug}

à Louisbourg Le 27. 7bre 1762

[...] quant à ma situation, elle est assez heureuse ; mon gendre pourra vous en faire le détail ; Sans la bibliomanie qui m'a repris je serois en argent, mais vous savez ce que c'est que cette maladie ; ma petite bibliotheque monte dejà à trois mille volumes tous bien choisis pour les matieres, et pour les editions. Comme j'espère determiner le duc dont je suis devenu le bibliothecaire avec cent Louis d'appointemens, à etablir une bibliotheque publique dont j'aurai l'inspection, je compte lui remettre mon cabinet tel qu'il est, et par là rattrapper environs douze mille francs qu'il me coute, et les placer pour en trouver le revenu au besoin.

\section{Ludwigsburg, den 27. September 1762}

[...] Mein Zustand ist ziemlich glücklich; mein Schwiegersohn wird Ihnen ausführliche Auskünfte geben. Wäre nicht meine Büchersucht, die mich wieder gepackt hat, hätte ich Geld. Sie wissen aber was diese Krankheit ist. Meine kleine Bibliothek zählt schon drei tausend Bände, alle, was den Inhalt und die Auflagen betrifft, gut ausgewählt. Da ich hoffe den Herzog, dessen Bibliothekar ich mit hundert Louis Gehalt geworden bin, dazu zu bewegen eine öffentliche Bibliothek zu gründen, worüber ich die Aufsicht haben werde, gedenke ich meine Sammlung ihm abzugeben so wie sie ist, und dabei ungefähr zwölf tausend francs, die sie mir kostet, zu kriegen, und das Geld anzulegen um im Notfall das Einkommen zu finden.

14) Am 11. Oktober 1753 schreibt Mme de Graffigny dem Freund Devaux: "Tu ne m'avois point dit du tout q[u]'Uriot fut en Lorraine" (Correspondance de Madame de Graffigny, Bd. 13 (2010), hrsg. von Diane Beelen Woody [u.a.], S. 102). Übersetzt: „Du hattest mir gar nicht gesagt, Uriot sei in Lothringen".

15) Batz, Karls-Schule, S. 107. In der Übersetzung (Description de l'Academie-Caroline) ist der Titel des Gedichtes angegeben (S. 133). Es wurde im Band 2 (1755) der Zeitschrift veröffentlicht (S. 254-260). In diesem Werk setzt sich Uriot mit der Ausbildung der Jugend auseinander.

16) Da Uriot nicht in Nancy wohnte, verkehrte er schriftlich mit der Akademie (Briefe, Aufsätze, Gedichte...).

17) Bibliothèque Stanislas, Signatur: 258721. Es gab auch einen einfachen Einband aus Papier. Die zwei Exemplare in Montbéliard (Mömpelgard) sind so eingebunden (Bibliothèque municipale de Montbéliard, Signatur: W 304 a und b. Die Signatur W bedeutet Württemberg).

18) Im Protokoll der Akademie wurde das alles eingetragen (Archives de I'Académie Stanislas de Nancy). In seinem Tagebuch schreibt Nicolas Durival, damals "lieutenant de police" in Nancy: "on avoit refusé d'admettre le S. Uriot, parce qu'il avoit été comédien" (Bibliothèque Stanislas, Manuskript 863, Band 6, BI. 78v; elektronische Ausgabe: journaldedurival.fr). Übersetzt: "Man hatte verweigert, den Herrn Uriot aufzunehmen, weil er Schauspieler gewesen war."

19) Bibliothèque Stanislas, Signatur: Rés. 10270. Uriot hat in diesem Exemplar handschriftliche Korrekturen eingetragen. Die Bayerische Staatsbibliothek bewahrt zwei Exemplare der Rede, das eine mit denselben Korrekturen wie in Nancy (Signatur: 4 Diss. 3621,15; digitalisiert), das andere ohne Korrekturen (Signatur: Germ. sp. 382, 30; digitalisiert). Im Exemplar von Montbéliard gibt es keine Korrekturen (Signatur: W 295). 
Uriot wollte wahrscheinlich das Geld anlegen, weil er 1762 drei kleine Kinder hatte: zwei Mädchen und einen Knaben, der im November 1761 geboren wurde. Eine zwanzigjährige Tochter war verheiratet. Beim Verkaufsprojekt seiner Sammlung dachte er wohl an das Geld, vielleicht aber auch an die Zukunft seiner Bücher: was hätten seine Erben damit gemacht? Als Bibliothekar der öffentlichen Bibliothek schenkte er dieser Anstalt Bücher, die er gekauft hatte (Herrmann, Uriot, S. 50). Anstatt "francs" hätte er auch "livres" schreiben können. In den Büchern des ehemaligen Kapuzinerklosters Belfort wurden die Preise in livres eingetragen. So weiß man zum Beispiel, dass die dreizehn Bände des Werkes "Abrégé de l'histoire ecclésiastique" (A Cologne, Aux dépens de la Compagnie, 17621767) 1776 insgesamt achtzig "livres" gekostet hatten (Bibliothèque municipale de Belfort, Fonds des Capucins, Signatur: $4^{\circ}$ 53).

\section{Handschriftliche Eintragungen}

1782 sendete Uriot nach Nancy "La Naissance de Félicité" und "Les Fêtes thessaliennes" mit handschriftlichen Korrekturen und Zusätzen (Bibliothèque Stanislas, Signatur: 80287-35 und 977). Bis jetzt wurden Eintragungen auch in folgenden Bibliotheken entdeckt: Bibliothèque Mazarine Paris (Korrekturen und Zusätze in "L'Amour fraternel" und "Les Fêtes thessaliennes". Sammelband, Signatur: $4^{\circ}$ 10918-125), Fürstlich Schaumburg-Lippische Hofbibliothek in Verwaltung des Niedersächsischen Landesarchivs, Standort Bückeburg, Historische Bibliothek der Stadt Rastatt, Bibliothèque municipale de Montbéliard (dieselben Korrekturen in "La Vérité telle qu'elle est". Bückeburg, Signatur: FHB, Gl 115; Rastatt, Signatur: P 584-BS; Montbéliard, zwei Exemplare, Signatur: W 147 a und b). In Montbéliard gibt es auch Widmungen und Korrekturen in "Description des fêtes", 1763 (zwei Exemplare, Signatur: W $212 \mathrm{c}$ und d). Es ist zu vermuten, dass Uriot die Exemplare durchsah, die für bestimmte Leser gedacht waren. Festgestellt wurde vor kurzem, dass die Exemplare der Bibliothèque Mazarine dem Prinz Xaver von Sachsen, der mit Carl Eugen verwandt war, gehörten.

Mariette Cuénin-Lieber 